\title{
Características físico-químicas e aceitação sensorial de salame tipo Italiano com adição de óleo de canola
}

\section{Physico-chemical characteristics and sensory acceptance of Italian- type salami with canola oil addition}

\author{
Ângela Maria Backes ${ }^{1}$; Nelcindo Nascimento Terra²; Liana Inês Guidolin Milani³; \\ Ana Paula de Souza Rezer³; Fernanda Luisa Lüdtke ${ }^{4}$; Carlos Pasqualin Cavalheiro ${ }^{1}$; \\ Leadir Lucy Martins Fries ${ }^{2 *}$
}

\begin{abstract}
Resumo
O objetivo deste trabalho foi avaliar os efeitos da substituição parcial da gordura suína por emulsão contendo óleo de canola em salame tipo Italiano. Foram elaborados três tratamentos, entre eles: Controle (100\% de gordura suína, sem substituição de gordura), T1 (15\% da gordura suína foi substituída pela emulsão contendo óleo de canola) e T2 (30\% da gordura suína foi substituída pela emulsão com óleo de canola). Foram avaliados as características físico-químicas dos salames $(\mathrm{pH}$, atividade de água, perda de peso, cor e oxidação lipídica) durante o período de fabricação e armazenamento, além da avaliação sensorial após o término do processamento. A adição do óleo de canola emulsionado em diferentes concentrações não afetou os valores de $\mathrm{pH}$ e cor durante o período de processamento, apesar de diferenças significativas encontradas nesses parâmetros durante o tempo de estocagem. A atividade de água não diferiu significativamente entre os tratamentos. No entanto, os tratamentos com adição de óleo de canola tiveram menor perda de peso do que o controle. Foi possível observar a elevação nos valores de oxidação lipídica no T2 durante o processamento e armazenamento dos embutidos, enquanto que no T1 os valores não diferiram do Controle ao final do processamento e mantiveram-se mais baixos que o controle durante o tempo de estocagem. Ainda, a substituição parcial da gordura suína por óleo de canola emulsionado não afetou a aceitação do produto quanto ao aroma, sabor, cor, textura e aparência visual. Dessa forma, a substituição de $15 \%$ de gordura suína por emulsão contendo óleo de canola em salames é uma alternativa viável para a diversificação de produtos.
\end{abstract}

Palavras-chave: Produto cárneo fermentado, óleo vegetal, armazenamento, oxidação lipídica

\footnotetext{
Abstract

The aim of this study was to evaluate the effects of partial pork fat replacement with emulsified canola oil in Italian-type salami. Three treatments were done: Control (100\% pork fat, without fat replacement), T1 (15\% pork fat was replaced by emulsified canola oil) and T2 (30\% pork fat was replaced by emulsified oil canola). There were evaluated the salamis' physicochemical characteristics ( $\mathrm{pH}$, water activity, weight loss, color and lipid oxidation) during the manufacture and storage period, and sensory

${ }^{1}$ Discentes do Curso de Pós-Graduação em Ciência e Tecnologia dos Alimentos, Universidade Federal de Santa Maria, UFSM, Santa Maria, RS. E-mail: angebackes@yahoo.com.br; cavalheiro.carlos@hotmail.com

${ }^{2}$ Profs. do Programa de Pós-Graduação em Ciência e Tecnologia dos Alimentos, UFSM, Santa Maria, RS. E-mail: nelcindo@terra. com.br; lucymicro@yahoo.com.br

${ }^{3}$ Técnicas de Laboratório do Dept ${ }^{\mathrm{o}}$ de Tecnologia e Ciência dos Alimentos, UFSM, Santa Maria, RS. E-mail: lianamilani@yahoo. com.br; anarezer@bol.com.br

${ }^{4}$ Discente do Curso de Graduação em Agronomia, UFSM, Santa Maria, RS. E-mail: felovatoludtke@gmail.com

* Autor para correspondência
} 
evaluation after the manufacture process. The emulsified canola oil addition at different levels did not change the $\mathrm{pH}$ and color during the manufacture process, even though significant differences were found in these parameters during the storage period. The water activity did not differ significantly among the treatments. However, the treatments with emulsified canola oil added have a lower weight loss than the control. It was possible to observe an elevation on lipid oxidation values in the T2 during manufacture and storage periods, while in the T1, the values did not differ from the control at the end of manufactures and remained lower than the control during the storage period. Even more, the partial replacement of pork fat by emulsified canola oil did not affect the acceptance of the product for aroma, flavor, color, texture and visual appearance. Thus, the $15 \%$ pork fat replacement by emulsified canola oil in Italian-type salami is a viable alternative for the product diversification.

Key words: Fermented meat product, vegetable oil, storage, lipid oxidation

\section{Introdução}

A produção de salames envolve três fases bem conhecidas: a mistura de ingredientes, fermentação e secagem (DEMEYER; VERPLAETSE; GISTELINCK, 1986). Mudanças físicas, microbiológicas e bioquímicas (FRANCO et al., 2002; GARCÍA-FONTAN et al., 2007), envolvendo enzimas da carne e enzimas microbianas, acontecem durante a fermentação e secagem. Essas mudanças são influenciadas pelas características da matéria prima (BACUS, 1984) e das condições de processo (SOYER; ERTAS; ÜZÜMCÜOGLU, 2005) e se refletem nas propriedades organolépticas finais do produto.

Os salames são produtos cárneos com alto conteúdo de gordura, que pode ser visualizada inclusive no produto fatiado. Geralmente, esse tipo de produto contém cerca de $30 \%$ de gordura, sendo $35 \%$ o máximo permitido pela legislação brasileira (BRASIL, 2000). A gordura presente contribui para o flavour, textura, suculência e sabor do produto, fatores que determinam a qualidade e aceitabilidade dos embutidos fermentados (WIRTH, 1988).

As crescentes preocupações dos consumidores sobre o risco de desenvolvimento de doenças, associado ao consumo de alimentos ricos em gorduras, principalmente a gordura saturada, levou a indústria alimentícia a criar novos produtos e a reformulação dos produtos tradicionais (MENDOZA et al., 2001; MUGUERZA et al., 2004; JIMÉNEZ-COLMENERO, 2007). Entre as estratégias para a redução de gordura saturada nos embutidos fermentados inclui o uso de óleos vegetais como substitutos da gordura animal, contribuindo para a elaboração de produtos com melhor perfil de ácidos graxos e menor nível de colesterol, quando comparado com um produto tradicional (ÖZVURAL; VURAL, 2008).

A ingestão de ácidos graxos insaturados, principalmente os da classe ômega-3, tem efeitos positivos na saúde do consumidor, já que contribuem para o decréscimo dos níveis de lipoproteínas de baixa densidade (LDL) e elevação das lipoproteínas de alta densidade (HDL), colaborando para a redução na incidência de doenças coronarianas (MENTE et al., 2009). Nesse contexto, o óleo de canola tem ganhado grande destaque, principalmente por promover benefícios à saúde do consumidor (McDONALD et al., 1989), pelo seu efeito hipocolesterêmico (NYDAHL et al., 1995), antitrombótico (KWON et al., 1991) e por possuir uma maior proporção de ácidos graxos insaturados para ácidos graxos saturados do que a gordura animal (ESKIN; McDONALD, 1991; LIU; HUFFMAN, EGBERT, 1991).

Vários estudos têm relatado o desenvolvimento de produtos cárneos com menor teor de gordura saturada, através da utilização de óleos vegetais, principalmente óleo de soja, linhaça, canola, girassol e azeite de oliva, como substitutos de gordura animal (MUGUERZA et al., 2004; LURUEÑAMARTINEZ; VIVAR-QUINTANA; REVILLA, 2004; PELSER et al., 2007; SANTOS et al., 2008; 
VALENCIA et al., 2008; DEL-NOBILE et al., 2009; CHOI et al., 2013; YUNES et al., 2013).

Dessa forma, o objetivo deste estudo foi avaliar os efeitos da substituição parcial da gordura suína por uma emulsão contendo óleo de canola em salame tipo Italiano, analisando as características físico-químicas e a aceitação sensorial durante os períodos de processamento e armazenamento dos embutidos.

\section{Materiais e Métodos}

\section{Elaboração dos salames}

Para melhorar a estabilidade do óleo de canola no produto cárneo fermentado, foi realizada a emulsão do óleo com água e proteína isolada de soja. O processo de emulsificação do óleo de canola foi realizado conforme Bloukas, Paneras e Fournitzis (1997), com modificações. A emulsão foi preparada através da mistura manual, de 4 partes de água com 1 parte de proteína isolada de soja $\left(90 \%\right.$ de teor proteico) $\left(\right.$ Bremil $\left.^{\circledR}\right)$, seguida da adição lenta de 6 partes de óleo de canola (Bunge ${ }^{\circledR}$ Alimentos). Essa emulsão foi mantida por um dia a temperatura ambiente até ser adicionada nas massas cárneas.

Os salames tipo Italiano foram elaborados de acordo com a formulação e procedimentos descritos por Terra (1998). Foram utilizados como ingredientes: carne suína, 65,00\%; carne bovina, 20,00\%; sal, 3,00\% $\left(\right.$ Synth $\left.^{\circledR}\right)$; glicose, 0,30\% $\left(\right.$ Synth $\left.^{\circledR}\right)$; sacarose, 0,20\% $\left(\right.$ Synth $\left.^{\circledR}\right)$; sais de cura, 0,30\% $\left(\right.$ Bremil $\left.^{\circledR}\right)$; pimenta moída, 0,20\% $\left(\right.$ Kitano $\left.^{\circledR}\right)$; alho em pó, $0,20 \% \quad\left(\operatorname{Knorr}^{\circledR}\right)$; noz moscada,
0,20\% (MasterFoods $^{\circledR}$ ); fixador de cor, $0,25 \%$ $\left(\right.$ Bremil $\left.^{\circledR}\right)$ e cultura starter comercial contendo os micro-organismos Pediococcus pentosaceus e Staphylococcus xylosus (Christian Hansen ${ }^{\circledR}$ ). Após o processo de moagem das carnes suína e bovina, foi realizada a mistura com os demais ingredientes, exceto a gordura animal. Posteriormente à mistura, a massa cárnea foi divida em três lotes de $5 \mathrm{~kg}$ cada, que deram origem aos três tratamentos. Em cada lote, foi acrescentada, manualmente, uma quantidade pré-definida de toucinho congelado, cortado em cubos (aproximadamente $1 \mathrm{~cm}^{3}$ ), além da emulsão com óleo de canola. Os tratamentos foram os seguintes: Controle (C) - 100\% de gordura suína e sem substituição pela emulsão com óleo de canola; Tratamento 1 (T1) - substituição de $15 \%$ de gordura suína por emulsão de óleo de canola; e, Tratamento 2 (T2) - substituição de $30 \%$ da gordura suína pela emulsão contendo óleo de canola. A massa cárnea foi embutida em tripas artificiais de colágeno $(60 \mathrm{~mm}$ de diâmetro x $15 \mathrm{~cm}$ de comprimento) e as peças foram encaminhadas para câmara de maturação (Menoncin ${ }^{\circledR}$, Erechim, Brasil), com temperatura e umidade relativa do ar controladas $\left(1^{\circ}\right.$ dia: temperatura de $25^{\circ} \mathrm{C} / 95 \%$ de umidade relativa; $2^{\circ}$ dia: $24^{\circ} \mathrm{C} / 93 \%$; $3^{\circ}$ dia: $23^{\circ} \mathrm{C} / 90 \%$; $4^{\circ}$ dia: $22^{\circ} \mathrm{C} / 85 \%$; $5^{\circ}$ dia: $21^{\circ} \mathrm{C} / 80 \% ; 6^{\circ}$ dia: $20^{\circ} \mathrm{C} / 75 \%$ e; $7^{\circ}$ até o $28^{\circ}$ dia: $18^{\circ} \mathrm{C} / 75 \%$ ). Após 28 dias, a atividade de água atingiu valores inferiores a 0,87 , sendo considerado o término do processo de fabricação dos salames (TERRA, 1998). As peças então, foram embaladas sob vácuo e armazenadas em temperatura ambiente $\left(20^{\circ} \mathrm{C}\right)$. $\mathrm{O}$ aspecto geral dos salames contendo emulsão com óleo de canola pode ser visualizado na Figura 1. 
Figura 1. Aspecto geral do salame tipo Italiano com substituição da gordura suína por emulsão contendo óleo de canola.

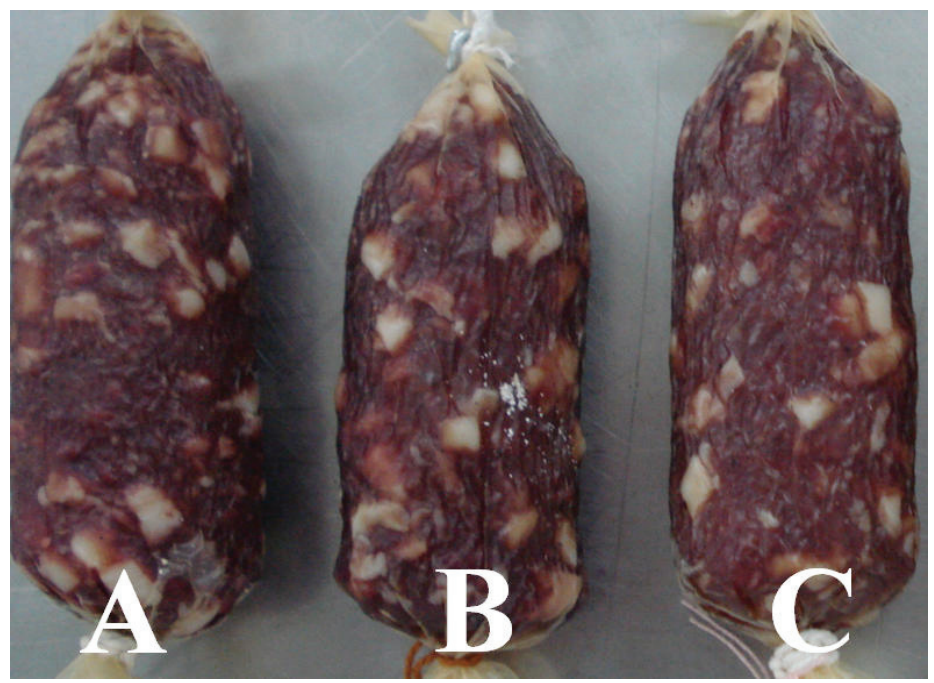

(A) Controle: sem substituição da gordura suína pela emulsão com óleo de canola; (B) T1: 15\% de substituição da gordura suína pela emulsão com óleo de canola; (C) T2: 30\% de substituição da gordura suína pela emulsão com óleo de canola.

Fonte: Elaboração dos autores.

\section{Análises físico-químicas}

As características físico-químicas ( $\mathrm{pH}$, atividade de água, perda de peso, cor e oxidação lipídica) dos salames adicionados de óleo de canola foram avaliados durante o período de fabricação e armazenamento. As análises de $\mathrm{pH}$ e cor foram executadas nos dias $0,5,7,14,21$ e 28 de fabricação e nos dias 30, 60 e 90 de armazenamento. A determinação da atividade de água e perda de peso foi realizada semanalmente durante os 28 dias de fabricação $(0,7,14,21$ e 28 dias). Devido à importância de monitorar a oxidação lipídica, além de ser realizado semanalmente durante o tempo de fabricação, o teste foi feito quinzenalmente durante o tempo de estocagem, ou seja, aos 30, 45, 60, 75 e 90 dias de armazenamento.

A determinação do $\mathrm{pH}$ foi realizada em potenciômetro digital modelo DM 22 (Digimed $^{\circledR}$, São Paulo, Brasil), de acordo com a metodologia proposta por Terra e Brum (1988). A atividade de água foi medida utilizando o aparelho $400 \mathrm{CE}$, (Testo ${ }^{\circledR}$ GMBH \& CO., Lenzkirch, Alemanha), por medida direta nas amostras. A avaliação da perda de peso foi determinada pela diferença de peso existente entre as peças cárneas no momento do embutimento e após o produto acabado (28 dias). A medição da cor foi realizada pelo colorímetro Minolta CR-300 (Minolta Chroma Meter ${ }^{\circledR}$, Osaka, Japão), expressando no sistema CIELAB os valores de luminosidade $\left(L^{*}\right)$, componente vermelho-verde $\left(a^{*}\right)$ e componente amarelo-zul (b*). As substâncias reativas ao ácido tiobarbitúrico (TBARS) resultantes da oxidação lipídica foram determinadas segundo a metodologia proposta por Raharjo, Sofos e Schmidt (1992).

\section{Análise sensorial}

Os diferentes tratamentos de salame também foram avaliados sensorialmente quanto a aceitação, utilizando uma escala hedônica estruturada de sete pontos, variando de desgostei muitíssimo (1) a gostei muitíssimo (7), conforme metodologia de Meilgaard, Civille e Carr (2006). Foram avaliados os parâmetros de cor, aroma, sabor, textura e aparência visual. O estudo foi aprovado pelo Comitê de Ética 
em Pesquisa (CEP) da Universidade Federal de Santa Maria (UFSM) (número 0109.0.243.000-10). A equipe foi composta por estudantes, professores e funcionários da universidade, todos consumidores de salame, sendo $52 \%$ do sexo masculino, $48 \%$ do sexo feminino, com idades entre 18 e 57 anos.

Os salames foram fornecidos aos provadores em fatias de aproximadamente $0,8 \mathrm{~cm}$, servidas em prato branco e codificadas aleatoriamente. Também foram fornecidos água e biscoito água e sal para limpeza do palato entre as amostras.

\section{Delineamento experimental e análise estatística}

O desenho experimental foi inteiramente casualizado, com três níveis de substituição de gordura $(0 ; 15 ; 30 \%)$, totalizando três unidades experimentais. Os dados das determinações físicoquímicas foram feitas em quintuplicatas, enquanto a análise sensorial participaram 35 julgadores consumidores de salames. Todos os dados foram avaliados por Análise de Variância (ANOVA), sendo que as médias foram comparadas pelo teste de Tukey, considerando o nível de significância de $5 \%(\mathrm{p}<0,05)$, utilizando o pacote estatístico SPSS (9.0, IBM SPSS - Statistical Package for the Social Sciences).

\section{Resultados e Discussão}

\section{Análises físico-químicas}

Foi possível observar a queda do $\mathrm{pH}$ nos primeiros dias e a sua elevação no final do período de processamento e durante o armazenamento de todos os embutidos (Figura 2a), sendo que essas oscilações são características da fabricação dos produtos cárneos fermentados. Essa queda nos valores de $\mathrm{pH}$ pode ser atribuída ao crescimento de bactérias láticas que fermentam os carboidratos presentes na carne (LÜCKE, 1994). Resultados similares já foram reportados em produtos curados fermentados (CAVALHEIRO et al., 2010; OLIVARES et al., 2010; LORENZO; FRANCO, 2012).

Os tratamentos contendo emulsão com óleo de canola (T1 e T2) não apresentaram alterações significativas no $\mathrm{pH}$ quando comparados com o controle (C) no final do processamento dos salames (28 dias). Porém, durante o armazenamento, os tratamentos $\mathrm{T} 1$ e $\mathrm{T} 2$ apresentaram $\mathrm{pH}$ menor que o controle. Diferente dos resultados obtidos neste trabalho, Severini, De Pilli e Baiano (2003) não encontraram diferença estatística após 30 dias de armazenamento de salames adicionados de emulsão com azeite de oliva.

Observou-se a redução gradativa na atividade de água dos embutidos fermentados durante o período de fabricação (Figura 2b). Esta queda pode ser atribuída ao decréscimo nos valores de $\mathrm{pH}$, pois a capacidade de retenção de água das proteínas da carne é diminuída quando o $\mathrm{pH}$ se aproxima do seu ponto isoelétrico (5,3) (TERRA, 1998), acelerando a desidratação e consequentemente reduzindo a atividade de água. Além disso, a incorporação do óleo de canola emulsionado não afetou esses valores, uma vez que este parâmetro não apresentou diferença estatística entre os tratamentos no final do processamento. Em estudos realizados por Olivares et al. (2010) com embutidos fermentados, utilizando diferentes concentrações de gordura suína, os valores de atividade de água no final do processamento (62 dias) também não apresentaram diferença estatística. Por outro lado, Severini, De Pilli e Baiano (2003) encontraram resultados diferentes aos obtidos neste experimento, já que as três formulações de salames com azeite de oliva tiveram valores significativamente superiores a formulação padrão, ao final de 60 dias de maturação. 
Figura 2. Efeitos da substituição da gordura suína por emulsão contendo óleo de canola sobre o pH (a), Atividade de água (b) e perda de peso (em \%) (c), de salame tipo Italiano durante o período de fabricação e armazenamento.

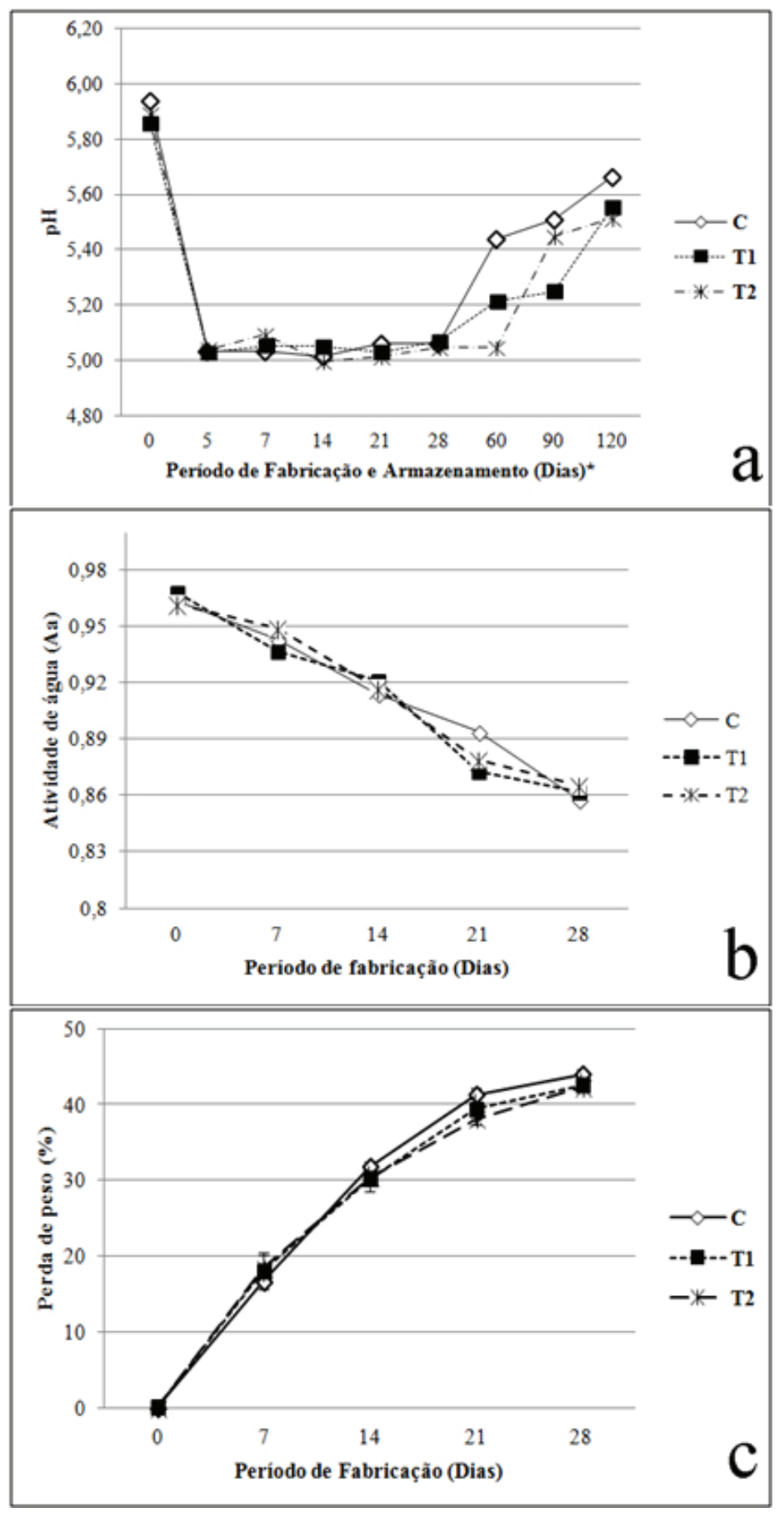

C: sem substituição da gordura suína pela emulsão com óleo de canola; T1: $15 \%$ de substituição da gordura suína pela emulsão com óleo de canola; T2: 30\% de substituição da gordura suína pela emulsão com óleo de canola.

* O período de fabricação foi considerado até o $28^{\circ}$ dia.

Fonte: Elaboração dos autores. 
A diminuição da quantidade de água dos embutidos é um dos principais responsáveis pela textura (FERNÁNDEZ et al., 2000) e fatiabilidade do produto final (BUCKENHÜSKES, 1993). Verificou-se que a incorporação da emulsão com óleo de canola em salames resultou, no final do período de fabricação, em perdas de peso de 42,54 e $42,27 \%$ para o $\mathrm{T} 1$ e $\mathrm{T} 2$, respectivamente, enquanto que o controle teve $44,00 \%$ de quebra de peso (Figura 2c). Apesar da diferenca estatistica entre os tratamentos com a incorporação de óleo de canola e o controle, todos os tratamentos apresentaram perda de peso acima de $40 \%$, considerado ideal para os produtos carneos fermentados secos (TERRA, 1998).

$\mathrm{Na}$ Tabela 1 apresentam-se os valores da cor instrumental dos produtos contendo emulsão com óleo de canola. Quando analisado o parâmetro luminosidade $\left(L^{*}\right)$, foi possível observar o decréscimo nos valores em todos os tratamentos durante o período de fabricação e armazenamento. Apesar de não apresentarem diferença estatística no inicio e final do processamento quanto a este parâmetro, ao final de 120 dias, além dos valores de luminosidade ser inferiores aos apresentados aos 28 dias, o produto $\mathrm{T} 1$ mostrou-se mais escuro que o controle, mas sem diferença do $\mathrm{T} 2$. Os maiores valores de atividade de água encontrados nos embutidos no primeiro dia de fabricação aumentaram a reflexão da luz sobre sua superfície, conferindo mais luminosidade e maior brilho às peças (MATOS et al., 2007). Com relação à intensidade de vermelho $\left(a^{*}\right)$, foi observado um comportamento variável em todas as formulações durante o período de fabricação e estocagem, mas com o aumento nos valores dos tratamentos $\mathrm{T} 1$ e $\mathrm{T} 2$ até o final do processamento e do armazenamento. Por outro lado, ocorreu a diminuição da intensidade de amarelo $\left(b^{*}\right)$, em todos os tratamentos, durante o processamento, mas com a posterior elevação nos resultados até o final do armazenamento dos salames. Essa queda nos valores de $b *$ durante a fermentação e a maturação é atribuída ao decréscimo no consumo de oxigênio pelos microrganismos e a consequente diminuição da oximioglobina, a qual contribui para a tonalidade amarela (PÉREZ-ALVAREZ et al., 1999). Os valores de $L^{*}$ e $b^{*}$ observados neste experimento são similares aos obtidos em Chorizo de Pamplona com a substituição da gordura suína por diversas concentrações de óleo de soja (MUGUERZA; ANSORENA; ASTIASARÁN, 2003). 
Tabela 1. Efeitos da substituição da gordura suína por emulsão contendo óleo de canola sobre a cor de salame tipo Italiano, expressa como $L^{*}$ (luminosidade), $a^{*}$ (componente vermelho-verde) e $b^{*}$ (componente amarelo-azul), durante o período de fabricação e armazenamento*.

\begin{tabular}{|c|c|c|c|c|}
\hline Dia & Tratamento & $L^{*}$ & $a^{*}$ & $b^{*}$ \\
\hline \multirow{3}{*}{$\mathbf{0}$} & $\mathbf{C}$ & $54,04 \pm 4,88^{\mathrm{a}}$ & $25,74 \pm 3,34^{\mathrm{a}}$ & $13,79 \pm 1,08^{\mathrm{a}}$ \\
\hline & T1 & $55,98 \pm 1,05^{\mathrm{a}}$ & $13,56 \pm 2,18^{\mathrm{c}}$ & $11,45 \pm 0,81^{\mathrm{b}}$ \\
\hline & T2 & $53,09 \pm 0,83^{\mathrm{a}}$ & $16,33 \pm 0,96^{\mathrm{b}}$ & $12,63 \pm 0,84^{\mathrm{a}}$ \\
\hline \multirow{3}{*}{5} & $\mathrm{C}$ & $49,16 \pm 1,03^{\mathrm{a}}$ & $19,49 \pm 0,50^{\mathrm{b}}$ & $10,68 \pm 0,48^{\mathrm{a}}$ \\
\hline & T1 & $49,96 \pm 0,82^{\mathrm{a}}$ & $22,08 \pm 0,66^{\mathrm{a}}$ & $10,74 \pm 0,34^{\mathrm{a}}$ \\
\hline & $\mathbf{T} 2$ & $44,20 \pm 0,63^{b}$ & $20,37 \pm 0,29^{b}$ & $10,52 \pm 0,44^{\mathrm{a}}$ \\
\hline \multirow{3}{*}{7} & C & $45,99 \pm 2,18^{\mathrm{a}}$ & $22,78 \pm 1,45^{\mathrm{a}}$ & $10,49 \pm 0,41^{\mathrm{a}}$ \\
\hline & T1 & $45,81 \pm 1.08^{\mathrm{a}}$ & $19,53 \pm 1,39^{b}$ & $8,01 \pm 0,36^{\mathrm{b}}$ \\
\hline & T2 & $47,12 \pm 0,91^{\mathrm{a}}$ & $18,58 \pm 0,93^{b}$ & $8,20 \pm 0,46^{\mathrm{b}}$ \\
\hline \multirow{3}{*}{14} & C & $40,83 \pm 1,50^{\mathrm{b}}$ & $19,84 \pm 0,48^{\mathrm{a}}$ & $6,86 \pm 0,19^{b}$ \\
\hline & T1 & $45,84 \pm 0,87^{\mathrm{a}}$ & $17,97 \pm 0,41^{\mathrm{b}}$ & $7,41 \pm 0,12^{\mathrm{ab}}$ \\
\hline & T2 & $42,93 \pm 1,68^{b}$ & $18,09 \pm 0,40^{\mathrm{b}}$ & $8,01 \pm 0,75^{\mathrm{a}}$ \\
\hline \multirow{3}{*}{21} & $\mathrm{C}$ & $43,05 \pm 0,96^{\mathrm{a}}$ & $19,89 \pm 0,76^{\mathrm{b}}$ & $6,72 \pm 0,40^{\mathrm{a}}$ \\
\hline & T1 & $42,45 \pm 0,75^{\mathrm{a}}$ & $18,41 \pm 0,71^{\mathrm{ab}}$ & $6,15 \pm 0,83^{\mathrm{a}}$ \\
\hline & T2 & $39,29 \pm 0,94^{\mathrm{b}}$ & $17,80 \pm 0,72^{\mathrm{bc}}$ & $6,12 \pm 0,10^{\mathrm{a}}$ \\
\hline \multirow{3}{*}{28} & C & $40,35 \pm 1,70^{\mathrm{a}}$ & $17,75 \pm 0,67^{\mathrm{a}}$ & $5,72 \pm 0,64^{\mathrm{ab}}$ \\
\hline & T1 & $41,33 \pm 1,90^{\mathrm{a}}$ & $16,61 \pm 0,72^{\mathrm{a}}$ & $5,04 \pm 0,43^{\mathrm{b}}$ \\
\hline & $\mathbf{T} 2$ & $41,98 \pm 0,88^{\mathrm{a}}$ & $17,30 \pm 0,83^{\mathrm{a}}$ & $6,61 \pm 0,62^{\mathrm{a}}$ \\
\hline \multirow{3}{*}{60} & $\mathrm{C}$ & $41,21 \pm 0,87^{\mathrm{a}}$ & $16,73 \pm 0,46^{\mathrm{e}}$ & $4,78 \pm 0,23^{\mathrm{e}}$ \\
\hline & T1 & $38,67 \pm 1,03^{\mathrm{b}}$ & $19,09 \pm 0,33^{\mathrm{a}}$ & $6,77 \pm 0,11^{\mathrm{a}}$ \\
\hline & $\mathbf{T} 2$ & $39,61 \pm 0,91^{\mathrm{b}}$ & $16,93 \pm 0,34^{b}$ & $6,33 \pm 0,09^{b}$ \\
\hline \multirow{3}{*}{90} & $\mathrm{C}$ & $39,23 \pm 1,25^{\mathrm{c}}$ & $19,70 \pm 0,63^{\mathrm{a}}$ & $8,01 \pm 0,44^{\mathrm{a}}$ \\
\hline & T1 & $40,23 \pm 0,98^{\mathrm{b}}$ & $18,17 \pm 0,18^{\mathrm{b}}$ & $7,46 \pm 0,12^{\mathrm{a}}$ \\
\hline & $\mathrm{T} 2$ & $41,42 \pm 0,71^{\mathrm{a}}$ & $16,63 \pm 0,74^{\mathrm{c}}$ & $7,64 \pm 0,21^{\mathrm{a}}$ \\
\hline \multirow{3}{*}{120} & C & $40,71 \pm 0,57^{\mathrm{a}}$ & $21,07 \pm 0,21^{\mathrm{a}}$ & $8,46 \pm 0,12^{\mathrm{b}}$ \\
\hline & T1 & $38,92 \pm 0,84^{b}$ & $21,56 \pm 0,59^{\mathrm{a}}$ & $9,45 \pm 0,32^{\mathrm{a}}$ \\
\hline & $\mathbf{T} 2$ & $39,54 \pm 0,39^{\mathrm{ab}}$ & $16,35 \pm 0,48^{\mathrm{b}}$ & $9,05 \pm 0,41^{\mathrm{ab}}$ \\
\hline
\end{tabular}

${ }^{a}$ Médias $(\mathrm{n}=5)$ acompanhadas por letras diferentes na mesma coluna e no mesmo dia apresentam diferença significativa pelo teste de Tukey.

C: sem substituição da gordura suína pela emulsão com óleo de canola; T1: 15\% de substituição da gordura suína pela emulsão com óleo de canola; T2: 30\% de substituição da gordura suína pela emulsão com óleo de canola.

*O período de fabricação foi considerado até o $28^{\circ}$ dia.

Fonte: Elaboração dos autores.

Em relação aos valores de TBARS nos salames contendo emulsão com óleo de canola (Figura 3), foi possível observar que durante os primeiros sete dias de fabricação, o T2 apresentou valores significativamente inferiores aos demais. No entanto, a partir do $14^{\circ}$ dia de processamento, o T2 passou a 
ter maior aumento nos valores de TBARS que o T1 e o Controle, diferindo estatisticamente dos demais tratamentos no final do processamento (28 dias), que apresentaram valores de $0,81 \mathrm{mg}$ de malonaldeído/ $\mathrm{Kg}$ para o controle, $0,91 \mathrm{mg}$ de malonaldeído/Kg para o $\mathrm{T} 1$ e 1,88 mg de malonaldeído/Kg para $\mathrm{T} 2$. De acordo com Connell (1990), o limite aceitável para a oxidação lipídica é de $2 \mathrm{mg}$ de malonaldeído/ $\mathrm{kg}$ de amostra. Dessa forma, conforme a Figura
3, o T2 atingiu esse valor no $75^{\circ}$ dia, o Controle atingiu no $90^{\circ}$ dia, enquanto o $\mathrm{T} 1$, não atingiu o limiar até o $120^{\circ}$ dia. A maior oxidação lipídica encontrada no $\mathrm{T} 2$ deve-se à maior quantidade de gordura insaturada no óleo de canola, favorecendo as reações com o oxigênio e influenciada por fatores como luz, calor e substâncias pró-oxidantes (SALLAM; SAMEJIMA, 2004; CHENG; WANG; OCKERMAN, 2007).

Figura 3. Efeitos da substituição da gordura suína por emulsão contendo óleo de canola sobre os valores de TBARS de salame tipo Italiano durante o período de fabricação e armazenamento.

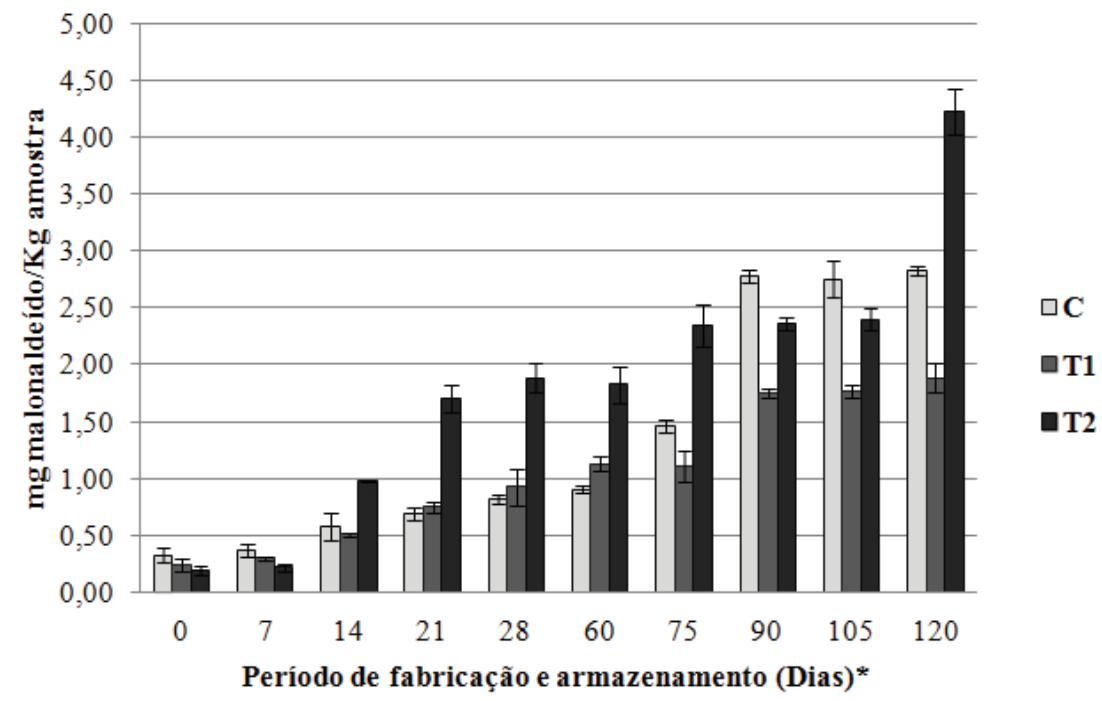

C: sem substituição da gordura suína pela emulsão com óleo de canola; T1: 15\% de substituição da gordura suína pela emulsão com óleo de canola; T2: 30\% de substituição da gordura suína pela emulsão com óleo de canola.

* O período de fabricação foi considerado até o $28^{\circ}$ dia.

Fonte: Elaboração dos autores.

Apesar do aumento da oxidação lipídica em todos os tratamentos durante o período de armazenamento, observou-se que aos 120 dias, todos os tratamentos apresentaram diferença estatística entre si, sendo que o menor valor de TBARS foi obtido pelo T1, seguindo do Controle e do T2. Portanto, a substituição de $30 \%$ de gordura suína por óleo de canola emulsionado afetou significativamente a oxidação lipídica dos salames durante o processamento e armazenamento. Embora o óleo de canola seja rico no antioxidante natural tocoferol (O'BRIEN, 2008), a presença de ácidos graxos insaturados, especialmente os poliinsaturados, colaboram para o aumento da suscetibilidade da oxidação lipídica (VALENCIA et al., 2008). Pelser et al. (2007) verificaram, durante 83 dias de armazenamento, que os valores de TBARS foram semelhantes entre o controle e os embutidos adicionados de óleo de canola préemulsionado, independente da quantidade de óleo. Além disso, no trabalho desenvolvido pelos mesmos autores, as amostras adicionadas de óleo de 
canola tiveram a formação de hexanal (produto da oxidação do ácido oléico) semelhante ao controle, demonstrando que o principal constituinte do óleo não foi degradado.

\section{Análise sensorial}

Não houve diferença de aceitação entre os tratamentos quanto aos parâmetros sensoriais analisados (Tabela 2). No geral foram observadas notas médias próximas de 5 para todos os tratamentos, demonstrando a aceitação dos salames pelos provadores. Bloukas, Paneiras e Fournitzis
(1997), em embutidos fermentados adicionados de azeite de oliva pré-emulsionado, também encontraram valores superiores a 4 (aceitável) para salames com adição de até $20 \%$ de óleo de oliva líquido e valores superiores a 5 (bom) para salames com até $20 \%$ de óleo de oliva pré-emulsionado. Porém, conforme visto por Del Nobile et al. (2009), maiores níveis de óleos vegetais adicionados (60 $100 \%$ ) em produtos fermentados podem acarretar em menor aceitabilidade pelos provadores, além de produtos com problemas tecnológicos (difícil fatiabilidade, separação da carne da tripa e baixa incorporação do óleo com a carne).

Tabela 2. Efeitos da substituição da gordura suína por emulsão contendo óleo de canola sobre as características sensoriais de salame tipo Italiano.

\begin{tabular}{lccc}
\hline \multirow{2}{*}{ Atributos } & & Tratamentos & \\
\cline { 2 - 4 } & Controle & T1 (15\%) & T2 (30\%) \\
\hline Sabor & $5,51 \pm 1,46^{\mathrm{a}}$ & $5,00 \pm 1,47^{\mathrm{a}}$ & $5,51 \pm 1,46^{\mathrm{a}}$ \\
Aroma & $4,91 \pm 1,46^{\mathrm{a}}$ & $4,85 \pm 1,31^{\mathrm{a}}$ & $5,25 \pm 1,29^{\mathrm{a}}$ \\
Cor & $5,60 \pm 1,35^{\mathrm{a}}$ & $5,28 \pm 1,15^{\mathrm{a}}$ & $5,88 \pm 0,83^{\mathrm{a}}$ \\
Textura & $5,48 \pm 0,98^{\mathrm{a}}$ & $5,28 \pm 0,98^{\mathrm{a}}$ & $5,42 \pm 1,14^{\mathrm{a}}$ \\
Aparência visual & $5,71 \pm 1,25^{\mathrm{a}}$ & $5,37 \pm 1,39^{\mathrm{a}}$ & $6,02 \pm 0,92^{\mathrm{a}}$ \\
\hline
\end{tabular}

${ }^{a}$ Médias ( $\left.n=35\right)$ acompanhadas por letras diferentes na mesma linha apresentam diferença significativa pelo teste de Tukey. Controle: sem substituição da gordura suína pela emulsão com óleo de canola; T1: 15\% de substituição da gordura suína pela emulsão com óleo de canola; T2: 30\% de substituição da gordura suína pela emulsão com óleo de canola.

Fonte: Elaboração dos autores.

\section{Conclusões}

As substituições de $15 \%$ e $30 \%$ de gordura suína por óleo de canola pré-emulsionado em salames acarretaram em alterações nos valores de $\mathrm{pH}$, perda de peso, níveis de oxidação e nos parâmetros de cor no final do processamento e durante o período de armazenamento, porém não comprometendo a aceitação sensorial dos produtos. Dessa forma, apesar das diferenças com o tratamento controle, conclui-se que a substituição de $15 \%$ de toucinho suíno por emulsão contendo óleo de canola permitiu a produção de salames diferenciados, visto que os atributos sensoriais foram mantidos, assim como a melhor estabilidade oxidativa do embutido durante o período de armazenamento.

\section{Agradecimentos}

Os autores agradecem ao $\mathrm{CNPq}$ pelo suporte financeiro e a CAPES pelas bolsas de estudo.

\section{Referências}

BACUS, J. Update: meat fermentation. Food Technology, Chicago, v. 38, n. 6, p. 59-63, 1984.

BLOUKAS, J. G.; PANERAS, E. D.; FOURNITZIS, G. C. Effect of replacing pork backfat with olive oil on processing and quality characteristics of fermented sausages. Meat Science, Barking, v. 45, n. 2, p. 133-144, 1997.

BRASIL. Ministério da Agricultura, Pecuária e do Abastecimento. Secretaria de Defesa Agropecuária. Instrução Normativa $n^{\circ}$. 22, de 31 de julho de 2000. 
Regulamentos técnicos de identidade e qualidade de salame tipo italiano. Diário Oficial [da] República Federativa do Brasil, Brasília, DF, 3 ago. 2000. Seção I, p. 15-28.

BUCKENHÜSKES, H. J. Selection criteria for lactic acid bacteria to be used as starter cultures for various food commodities. FEMS Microbiology Reviews, Oxford, v. 12, n. 1-3, p. 253-271, 1993.

CAVALHEIRO, C. P.; TERRA, N. N.; FRIES, L. L. M.; MILANI, L. I. G.; REZER, A. P. S.; CAVALHEIRO, C. V.; MANFIO, M. Características físico-químicas de embutido curado fermentado com adição de carne de avestruz associada à de suíno. Ciência Rural, Santa Maria, v. 40, n. 2, p. 447-452, 2010.

CHENG, J.; WANG, S.; OCKERMAN, H. W. Lipid oxidation and color change of salted pork patties. Meat Science, Barking, v. 75, n. 1, p. 71-77, 2007.

CHOI, Y. S.; PARK, K. S.; KIM, H. W.; HWANG, K. E.; SONG, D. H.; CHOI, M. S.; LEE, S. Y.; PAIK, H. D.; KIM, C. J. Quality characteristics of reduced-fat frankfurters with pork fat replaced by sunflower oils and dietary fiber extracted from makgeolli lees. Meat Science, Barking, v. 93, n. 3. p. 652-658, 2013.

CONNELL, J. J. Methods of assessing and selecting for quality. In: CONNELL, J. J. (Ed.). Control of fish quality. 3. ed. Berlin: Springer, 1990. p. 122-150.

DEL NOBILE, M. A.; CONTE, A.; INCORANATO, A. L.; PANZA, O.; SEVI, A.; MARINO, R. New strategies for reducing the pork back-fat content in typical Italian salami. Meat Science, Barking, v. 81, n. 1, p. 263-269, 2009.

DEMEYER, D. I.; VERPLAETSE, A.; GISTELINCK, M. Fermentation of meat: an integrated process. Food Chemistry Biotechnology, Bruxelas, v. 41, n. 5, p. 131140, 1986.

ESKIN, N. A. M.; McDONALD, B. E. Canola oil. Nutrition Bulletin, Londres, v. 16, n. 3, p. 138-146, 1991.

FERNÁNDEZ, M.; ORDÓÑEZ, J. A.; BRUNA, J. M.; HERRANZ, B.; HOZ, L. Accelerated ripening of dry fermented sausages. Trends in Food Science \& Technology, Amsterdam, v. 11, n. 6, p. 201-209, 2000.

FRANCO, D.; PRIETO, B.; CRUZ, J. M.; CARBALLO, J. Study of the biochemical changes during the processing of Androlla, a Spanish dry-cured pork sausage. Food Chemistry, Oxford, v. 78, n. 3, p. 339-345, 2002.

GARCÍA-FONTAN, M. C.; LORENZO, J. M.; PARADA, A.; FRANCO, I.; CARBALLO, J. Microbial characteristics of "androlla", a Spanish traditional pork sausage. Food Microbiology, Londres, v. 24, n. 1, p. 52-58, 2007.
JIMÉNEZ-COLMENERO, F. Healthier lipid formulation approaches in meat-based functional foods. Technological options for replacement of meat fats by non-meat fats. Trends in Food Science \& Technology, Amsterdam, v. 18, n. 1, p. 567-578, 2007.

KWON, J. S.; SNOOK, J. T.; WARDLAW, G. H.; HWANG, D. H. Effects of diets high in saturated fatty acids, canola oil or safflower oil on platelet function, thromboxane $\mathrm{B} 2$ formation, and fatty acid composition of platelet phospholipids. The American Journal of Clinical Nutrition, Bethesda, v. 54, n. 2, p. 351-358, 1991.

LIU, M. N.; HUFFMAN, D. L.; EGBERT, W. R. Replacement of beef fat with partially hydrogenated plant oil in lean ground beef patties. Journal of Food Science, Chicago, v. 56, n. 3, p. 861-862, 1991.

LORENZO, J. M.; FRANCO, D. Fat effect on physicochemical, microbial and textural changes through the manufactured of dry-cured foal sausage lipolysis, proteolysis and sensory properties. Meat Science, Barking, v. 92, n. 4, p. 704-714, 2012.

LÜCKE, F. K. Fermented meat products. Food Research International, Ontario, v. 27, n. 3, p. 299-307, 1994.

LURUEÑA-MARTÍNEZ, M. A.; VIVAR-QUINTANA, A. M.; REVILLA, I. Effect of locust bean/xanthan gum addition and replacement of pork fat with olive oil on the quality characteristics of low-fat frankfurters. Meat Science, Barking, v. 68, n. 3, p. 383-389, 2004.

MATOS, R. A.; MENEZES, C. M.; RAMOS, E. R.; RAMOS, A. L. S.; GOMIDE, L. A. M. Efeito do tipo de fermentação na qualidade final de embutidos fermentados cozidos elaborados a base de carne ovina. Boletim Centro de Pesquisa de Processamento de Alimentos, Curitiba, v. 25, n. 2, p. 225-234, 2007.

McDONALD, B. E.; GERRARD, J. M.; BRUCE, V. M.; CORNER, E. J. Comparison of the effect of canola oil and sunflower oil on plasma lipids and lipoproteins and on in vivo thromboxane A2 and prostacyclin production in health young men. The American Journal of Clinical Nutrition, Bethesda, v. 50, n. 6, p. 1382-1388, 1989.

MEIlgaARD, M.; CIVILle, G. V.; CARR, B. T. Sensory evaluation techniques. 4. ed. Bota Raton: CRC Press, 2006. 448 p.

MENDOZA, E.; GARCÍA, M. L.; CASAS, C.; SELGAS, M. D. Inulin as fat substitute in low fat, dry fermented sausages. Meat Science, Barking, v. 57, n. 4, p. 387-393, 2001.

MENTE, A.; KONING, L.; SHANNON, H. S.; ANAND, S. S. A systematic review of the evidence supporting a causal link between dietary factors and coronary heart disease. Archives of Internal Medicine, Londres, v. 169, n. 7, p. 659-569, 2009. 
MUGUERZA, E.; ANSORENA, D.; ASTIASARÁN, I. Improvement of nutritional properties of Chorizo de Pamplona by replacement of pork backfat with soy oil. Meat Science, Barking, v. 65, n. 4, p. 1361-1367, 2003.

MUGUERZA, E.; GIMENO, D.; ANSORENA, D.; ASTIASARÁN, I. New formulations for healthier dry fermented sausages: a review. Trends in Food Science \& Techonology, Amsterdam, v. 15, n. 9, p. 452-457, 2004.

NYDAHL, M.; GUSTAFSSON, I. B.; OHRVALL, M.; VESSBY, B. Similar effects of rapeseed oil (canola oil) and olive oil in a lipid-lowering diet for patients with hyperlipoproteinemia. Journal of The American College of Nutrition, Clearwater, v. 14, n. 6, p. 643-651, 1995.

O'BRIEN, R. D. Formulating and processing for applications. 3. ed. Lancaster: Technomic Publishing Company, 2008. 680 p.

OLIVARES, A.; NAVARRO, J. L.; SALVADOR, A.; FLORES, M. Sensory acceptability of slow fermented sausages based on fat content and ripening time. Meat Science, Barking, v. 86, n. 2, p. 251-257, 2010.

ÖZVURAL, E. B.; VURAL, H. Utilization of interesterified oil blends in the production of frankfurters. Meat Science, Barking, v. 78, n. 3, p. 211-216, 2008.

PELSER, W. M.; LINSSEN, J. P.; LEGGER, A.; HOUBEN, J. H. Lipid oxidation in n-3 fatty acid enriched Dutch style fermented sausages. Meat Science, Barking, v. 75, n. 1, p. 1-11, 2007.

PÉREZ-ALVAREZ, J. A.; SAYAS-BARBERÁ, M. E.; FERNÁNDEZ-LÓPEZ, J.; ARANDA-CATALÁ, V. Physicochemical characteristics of Spanish-type drycured sausage. Food Research International, Ontario, v. 32, n. 9, p. 599-607, 1999.

RAHARJO, S.; SOFOS, J. N.; SCHMIDT, G. R. Improved speed, specifity, and limit of determination of an aqueous acid extraction thiobarbituric acid - $\mathrm{C}_{18}$ method for measuring lipid peroxidation in beef. Journal of Agricultural and Food Chemistry, Washington, v. 40, n. 11, p. 2182-2185, 1992.
SALLAM, K. I.; SOMEJIMA, K. Microbiological and chemical quality of ground beef treated with sodium lactate and sodium chloride during refrigerated storage. LWT - Food Science and Technology, Oxford, v. 37, n. 8, p. 865-871, 2004.

SANTOS, C.; HOZ, L.; CAMBERO, M. I.; CABEZA, M. C.; ORDÓÑEZ, J. A. Enrichment of dry-cured ham with $\alpha$-linonelic acid and $\alpha$-tocopherol by the used of linseed oil and $\alpha$-tocopheryl acetate in pig diets. Meat Science, Barking, v. 80, n. 3, p. 668-674, 2008.

SEVERINI, C.; DE PILLI, T.; BAIANO, A. Partial substitution of pork backfat with extra-virgin olive oil in 'salami' products: effects on chemical, physical and sensorial quality. Meat Science, Barking, v. 64, n. 3, p. 323-331, 2003.

SOYER, A.; ERTAS, A. H.; ÜZÜMCÜOGLU, Ü. Effect of processing conditions on the quality of naturally fermented Turkish sausages (sucuks). Meat Science, Barking, v. 69, n. 1, p. 135-141, 2005.

TERRA, N. N. Apontamentos de tecnologia de carnes. São Leopoldo: Unisinos, 1998. 216 p.

TERRA, N. N.; BRUM, M. A. R. Carne e seus derivados - técnicas de controle de qualidade. São Paulo: Nobel, $1988.119 \mathrm{p}$.

VALENCIA, I.; O'GRADY, M. N.; ANSORENA, D.; ASTIASARÁN, I.; KERRY, J. P. Enhancement of the nutritional status and quality of fresh pork sausages following the addition of linseed oil, fish oil and natural antioxidants. Meat Science, Barking, v. 80, n. 4, p. 10461054, 2008.

WIRTH, F. Technologies for making fat-reduced meat products. Fleischwirtsch, Frankfurt, v. 68, n. 9, p. 1153 1156, 1988.

YUNES, J. F. F.; TERRA, N. N.; CAVALHEIRO, C. P.; FRIES, L. L. M.; GODOY, H. T.; BALLUS, C. A. Perfil de ácidos graxos e teor de colesterol de mortadela elaborada com óleos vegetais. Ciência Rural, Santa Maria, v. 43, n. 5, p. 924-929, 2013. 